\title{
THE CSO-JCMT SUBMILLIMETER INTERFEROMETER
}

\author{
J. E. CARLSTROM
}

Division of Physics, Math. \& Astronomy, Caltech, Pasadena, CA 91125

R. E. HILLS AND O. P. LAY

Mullard Radio Astronomy Observatory, Cambridge, England

\author{
B. FORCE, C. G. HALL, T. G. PHILLIPS, AND A. E. SCHINCKEL \\ CSO and JCMT Observatories, Hawaii
}

\begin{abstract}
A linked submillimeter-wave interferometer using the CSO $10.4 \mathrm{~m}$ and the JCMT $15 \mathrm{~m}$ telescopes located on Mauna Kea at an elevation of $4200 \mathrm{~m}$, has been built and tested successfully. The capabilities of the instrument and an outline of the type of observations that can be made with a single, fixed-baseline interferometer are given. In particular, an experiment to detect gas infalling onto low-mass protostellar sources is proposed. The design of the instrument is described briefly and the successful results of the first tests are summarized.
\end{abstract}

\section{INTRODUCTION}

High angular resolution observations at submillimeter wavelengths will enhance a wide spectrum of astronomical studies. Observations of disks around young stars are crucial to our understanding of star and planetary formation. Diameters of $\sim 100$ A.U., which corresponds to $\sim 1^{\prime \prime}$ for the closest candidates, are predicted. Probing the dusty cores of active galaxies also demands arcsecond resolution. The results from the millimeter arrays demonstrate that nearly all observational programs have benefitted from higher resolution (see reviews of BIMA, IRAM, NRO and OVRO in this volume). The higher dust and molecular line opacities and the higher energy levels at submillimeter wavelengths make high resolution submillimeter observations especially promising. The expected enhancement to submillimeter astronomy by interferometric techniques has motivated the development of the Smithsonian submillimeter array. Also driven by the unique and important astronomical observations that can be obtained, we have built a submillimeter-wave interferometer by linking the CSO $10.4 \mathrm{~m}$ and JCMT $15 \mathrm{~m}$ submillimeter telescopes located on Mauna Kea at $4200 \mathrm{~m}$. The instrument also provides the opportunity to address a number of technical issues facing submillimeter interferometry, such as atmospheric phase variations, and can be used as a test-bed for evaluating methods for overcoming them. 


\section{CAPABILITIES}

The CSO-JCMT interferometer combines the two largest existing submillimeter telescopes. The large collecting area, the low atmospheric opacity at Mauna Kea, and the high quality of the receivers available at both observatories, leads to impressive sensitivity. Here we give estimates of the single sideband sensitivity at $870 \mu \mathrm{m}(345 \mathrm{GHz})$ under good weather conditions. A $1000 \mathrm{~s}$ integration period is used in the calculations. Note, however, that due to the spatial filtering of an interferometer, the natural fringe rate, and the rapid phase-switching, the sensitivity of an interferometer is limited by how long one is willing to integrate, rather than by systematics as is usually the case with single dish observations. For continuum observations

$$
\Delta \mathrm{S}_{\nu}=16 \mathrm{mJy}\left(\frac{t_{\text {int }}}{1000 \mathrm{~s}}\right)^{-0.5}\left(\frac{\Delta \nu}{1 \mathrm{GHz}}\right)^{-0.5}
$$

and for spectral line observations

$$
\Delta \mathrm{S}_{\nu}=150 \mathrm{mJy}\left(\frac{\mathrm{t}_{\text {int }}}{1000 \mathrm{~s}}\right)^{-0.5}\left(\frac{\Delta v}{10 \mathrm{~km} \mathrm{~s}^{-1}}\right)^{-0.5}
$$

where $500 \mathrm{~K}$ single sideband system temperatures and $50 \%$ aperture efficiencies are used.

The $165 \mathrm{~m}$ baseline (nearly east-west) sets a resolution limit of

$$
\theta_{\min } \approx \frac{\lambda}{2|\mathbf{B}|}=0.6^{\prime \prime}\left(\frac{\lambda}{870 \mu \mathrm{m}}\right)
$$

Although lower resolution is possible due to the foreshortening of the baseline $\left(7^{\prime \prime}\right.$ is the shadowing limit), due to the fixed baseline this will not be appreciable for most sources, and about $1^{\prime \prime}$ will be typical a.t $\lambda=870 \mu \mathrm{m}$ or $0.3^{\prime \prime}$ at $300 \mu \mathrm{m}$, the shortest wavelength that the atmosphere and telescopes will permit.

Since we are mainly interested in thermal emission, we now consider the brightness sensitivity in the highest resolution element. For continuum observations

$$
\Delta \mathrm{T}_{\mathrm{B}}=0.7 \mathrm{~K}\left(\frac{\mathrm{t}_{\text {int }}}{1000 \mathrm{~s}}\right)^{-0.5}\left(\frac{\Delta \nu}{1 \mathrm{GHz}}\right)^{-0.5}
$$

and for spectral line

$$
\Delta \mathrm{T}_{\mathrm{B}}=6.0 \mathrm{~K}\left(\frac{\mathrm{t}_{\text {int }}}{1000 \mathrm{~s}}\right)^{-0.5}\left(\frac{\Delta v}{10 \mathrm{~km} \mathrm{~s}^{-1}}\right)^{-0.5}
$$

These sensitivities are promising when one considers that the typical dust and gas temperatures of embedded objects, circumstellar disks, and galactic nuclei are greater than $40 \mathrm{~K}$.

The brightness sensitivity of the interferometer at a particular frequency is easily estimated by scaling the single dish main beam sensitivities by the beam dilution factor which is $\leq 1000$ and is independent of observing frequency. 


\section{ASTRONOMICAL OBSERVATIONS WITH A SINGLE, FIXED- BASELINE INTERFEROMETER}

The single, fixed baseline severely limits the imaging capabilities of the interferometer, although one can improve the $u v$ coverage for continuum observations by using frequency synthesis techniques. Nevertheless, interesting and rewarding observations are possible. There are many sources for which a measurement of the flux from the central arcsecond is extremely important; these include accretion disks, the galactic center source SGR $A^{*}$, and the nuclei of IR luminous galaxies. For these objects measurements of the emission from the centrally concentrated gas and dust are urgently needed to constrain and distinguish between competing theories. The excellent observing capabilities at each telescope allow us to measure the single-dish (zero spacing) flux for the objects of interest, and the flux of the point sources which are used to calibrate the interferometer gain, thereby providing an accurate measure of the fractional flux detected at arcsecond scales by the interferometer.

Accurate relative positions of maser features from $\mathrm{H}_{2} \mathrm{O}, \mathrm{CH}_{3} \mathrm{OH}$, hydrogen recombination lines, and other species can be used to probe the the kinematics on sub-arcsecond scales (eg. Plambeck, Wright, and Carlstrom 1990; Planesas, Martin-Pintado, and Serabyn 1992). These types of measurements could also be made with thermal spectral line data from circumstellar disks, although with significantly less signal to noise, and therefore less accuracy.

Absorption line experiments are perhaps the most exciting, and unique observations which the interferometer enables. Existing arrays have shown the value of absorption observations toward high brightness HII regions or quasars (Welch et al. 1987; Marscher et al. 1991). The lines are seen in absorption since the continuum brightness is high in the small synthesized beam and the large scale emission is suppressed by the filtering property of the interferometer. Such experiments with a submillimeter interferometer are particularly interesting since the necessary brightness can be produced by the dust emission from low-mass protostellar sources. The detection of red-shifted absorption will provide the long sought, unambiguous signature of gas gravitationally collapsing onto a protostellar candidate.

\section{DESIGN OF THE CSO-JCMT INTERFEROMETER}

The design takes advantage of the existing instrumentation at each observatory. This includes the telescopes, receivers, and the JCMT spectrometer (the DAS) which can be configured to obtain cross-correlations. We installed a cable between the telescopes which enclosed four optical fibers. The cable threads through both telescope cable wraps and an underground conduit connecting the observatories. The optical fiber is terminated at each receiver cabin in commercial wideband ( $10 \mathrm{GHz}$ bandwidth) optical fiber receivers and transmitters (Ortel Corporation); there are no breaks or connectors in the optical fibers between the telescopes. A high spectral purity tone at $\sim 6 \mathrm{GHz}$ transmitted via the optical link provides the reference for the local oscillator (LO). This tone is generated by multiplying the output of an high quality synthesizer (an HP 8662 ). Since the optical fiber link has a noise floor of about $-125 \mathrm{dBc}$, a YIG 
oscillator is locked to the reference tone with about a $80 \mathrm{kHz}$ loop bandwidth to reject broadband noise; the YIG spectrum is cleaner than the transmitted spectrum at offset frequencies greater than $\sim 80 \mathrm{kHz}$. The tone frequency was chosen for maximum signal to noise of the phase locked LO (the multiplied output of a W-band Gunn oscillator) and maximum coherence. Degradation of the signal to noise by $20 \log (\mathrm{N})$ after multiplication by a factor of $\mathrm{N}$ suggests that one should transmit the reference tone at the highest possible frequency. However, the optical link noise increases in a non-linear manner with the carrier frequency. We found the best compromise to be about $6 \mathrm{GHz}$. We also transmit, on the same fiber as the LO reference, a $640 \mathrm{MHz}$ tone (obtained from the internal oscillator of the HP 8662 passed through a band pass filter) which is then used to phaselock a $10 \mathrm{MHz}$ crystal oscillator with about a $5 \mathrm{~Hz}$ loop bandwidth. Although an interruption in the $10 \mathrm{MHz}$ phaselock will result in a phase jump of $2 \pi n / 64$ where $n$ is an integer, in practice maintaining lock has not been a problem.

The resulting interreceiver phase jitter was measured by comparing an artificial fringe - obtained by mixing a $345 \mathrm{GHz}$ signal received from a source that illuminated both telescopes from a nearby ridge - with the $10 \mathrm{MHz}$ reference. The phase jitter was estimated to be a few degrees rms, confirming our bench results.

Two direct-digital-synthesizers (DDS), one to offset the first LO and the other to offset the IF, were used to compensate for the natural fringe rate at the center frequency of one sideband (selectable) while inserting a $2 \mathrm{MHz}$ artificial fringe rate in the opposite sideband; the correlator is thus sensitive to only one sideband. A coarse fiber optic delay-line is used so that the decorrelation is less than $1 \%$ in a short $(<10 \mathrm{~s})$ integration over a small channel width $(\leq 1.25 \mathrm{MHz})$ placed at any IF. The fine delay is then performed in software. The unique delay system is described in Lay, Hills, and Carlstrom (this volume).

Finally the correlations are performed by the flexible DAS; up to $1 \mathrm{GHz}$ bandwidth can be spanned with 800 independent channels. The two output buffers of the correlator were used synchronously with $180^{\circ}$ phase switching of the IF DDS to remove any offsets.

\section{RESULTS AND FUTURE PLANS}

The interferometer was first tested on 1992 June $15-16$. After verifying the that instrumentation worked on the artificial source, we observed water maser sources at $0.93 \mathrm{~mm}$ and detected the evolved star U Her, though not consistently. Poor weather and the short time period did not permit further progress.

Our second test period consisted of five nights over 1992 August 31 September 7. The weather was again poor, but this time we observed at $1.3 \mathrm{~mm}$. Due to the longer test period and better atmospheric opacity at $1.3 \mathrm{~mm}$ we were able to make considerable progress. After debugging the system, consistent detections of maser emission were routine. The vector averaged spectrum of $\mathrm{H}_{30 \alpha}$ maser emission from MWC 349 is shown in Figure 1. The delay lines and fine software delay worked well and allowed us to make consistent detections of continuum point sources (quasars). We were able to track the phase of a point sources and masers for several hours. This data was used to make a rough 


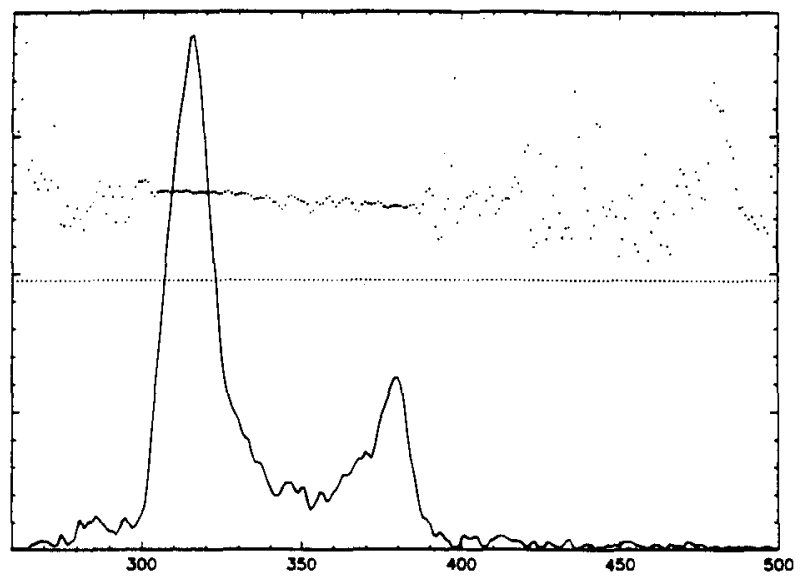

FIGURE I The vector averaged, cross-correlation spectrum of $\mathrm{H}_{30 \alpha}$ maser emission from MWC 349 at $1.29 \mathrm{~mm}$ taken with the CSO-JCMT interferometer. The dots mark the phase of each channel on a scale of $-\pi$ to $\pi$ which covers the upper half of the plot. The solid line connects the amplitude points. The full amplitude scale is $\sim 125 \mathrm{Jy}$. Each channel is $0.625 \mathrm{MHz}\left(0.81 \mathrm{~km} \mathrm{~s}^{-1}\right)$ wide.

astronomical determination of the baseline.

Although there are still many tests of the instrument left to make, and analysis of the existing data is still in progress, the first tests are extremely encouraging. Furthermore, they show that, weather permitting, we are ready to make many of the proposed astronomical submillimeter interferometry observations (in the $800 \mu \mathrm{m}$ and $600 \mu \mathrm{m}$ atmospheric windows) during our next session.

\section{ACKNOWLEDGMENTS}

We thank Mary Fuka, Chris Mayer and Ken Young for software support, Steve Padin, Marty Levin, Colin Masson and Doug Thornton for many useful discussions, Carl Johnson and James Anderson for surveying the baseline, and the entire JCMT and CSO staffs for their support. The CSO is supported by NSF grant AST 90-15755. The JCMT is operated by the Royal Observatory Edinburgh for the Science and Engineering Research Council of the U. K., the Canadian N. R. C., and the Netherlands Foundation for Research in Astronomy.

\section{REFERENCES}

Marscher, A. P, Bania, T. M., Wang, Z. 1991 ApJ, 371, L77.

Plambeck, R. L., Wright, M. C. H., and Carlstrom, J. E. 1990, ApJ, 348, L65.

Planesas, P., Martin-Pintado, J., Serabyn, E. 1992, ApJ, 386, L23

Welch, W. J., Dreher, J. W., Jackson, J. M., Terebey, S., and Vogel, S. N. 1987, Science, 238, 4833. 


\section{DISCUSSION}

H. Zinnecker As for the detection of protostellar infall, how strong a continuum source do you need in order to do your proposed absorption line experiment?

J. Carlstrom At the highest resolution at $345 \mathrm{GHz}, \sim 0.7^{\prime \prime}$, one Jy corresponds to a brightness of $\sim 30 \mathrm{~K}$. Therefore the experiment should be possible for sources with more than $\sim 1-2$ Jy unresolved by the interferometer. 